\title{
Osmophobia and allodynia are critical factors for suicidality in patients with migraine
}

\author{
Sung-Pa Park ${ }^{1 *}$, Jong-Geun Seo ${ }^{1}$ and Won-Kee Lee ${ }^{2}$
}

\begin{abstract}
Background: Sensory hypersensitivities are common phenomena in migraine. We examined the role of sensory hypersensitivities on suicidality in patients with migraine.

Methods: Patients with migraine (with or without aura) were consecutively recruited from our headache clinic. We asked them if they experienced photophobia, phonophobia, osmophobia, and allodynia during migraine attack. The Mini International Neuropsychiatric Interview was used to diagnose current major depressive disorder (MDD), current generalized anxiety disorder (GAD) and suicidality.

Results: Among 220 subjects, $25.5 \%$ had current MDD, $17.3 \%$ had current GAD, and $31.8 \%$ had suicidality. Patients with suicidality were more like to have a low household income, chronic migraine (CM), medication overuse headache, high headache intensity, osmophobia, allodynia, high disability, MDD, and GAD than those without suicidality. The strongest risk factor for suicidality by multivariate analyses was osmophobia (adjusted Odds Ratio [AOR] 3.12, $95 \%$ confidence interval [Cl] 1.57-6.21, $p=0.001$ ), followed by current MDD (AOR 2.99, $95 \% \mathrm{Cl}$ 1.33-6.76, $p=0.008$ ), CM (AOR 2.48, $95 \% \mathrm{Cl} 1.21-5.09, p=0.013$ ), current GAD (AOR 3.11, $95 \% \mathrm{Cl} 1.22-7.91, p=0.017$ ), and allodynia (AOR $2.72,95 \% \mathrm{Cl} 1.19-6.21, p=0.018$ ).
\end{abstract}

Conclusions: Osmophobia and allodynia are critical factors for suicidality in patients with migraine, after controlling for depression, anxiety, and CM.

Keywords: Suicide; Osmophobia; Allodynia; Migraine; Risk factor; Depression; Anxiety

\section{Background}

Adolescent and adult patients with migraine have been known to have a high risk of suicidality including suicidal ideation and attempt [1-6]. Risk factors for developing suicide in patients with migraine were psychiatric disorders [1-6], female gender [2], migraine with aura [1-3], higher headache frequency [3] and pain intensity $[4,6]$, younger age [5], unmarried state [5], and higher disability [5]. Among them, migraine with aura has been chosen as a migraine-specific factor for suicide after controlling for age, gender, major depression, and anxiety disorders [1-3].

Sensory hypersensitivities such as photophobia, phonophobia, osmophobia, and allodynia were frequently observed in patients with migraine [7-12]. Although the

\footnotetext{
* Correspondence: sppark@mail.knu.ac.kr

'Department of Neurology, School of Medicine, Kyungpook National University, 680 Gukchaebosang-ro, Jung-gu, Daegu 700-842, Republic of Korea

Full list of author information is available at the end of the article
}

frequency of allodynia is widely variable ( $15.1 \%$ to $69.7 \%$ ) [10-12], the frequencies of photophobia, phonophobia, and osmophobia were in the range from $43 \%$ to $86 \%[7-9,11,12]$. Recently, a hospital-based study in Taiwan reported that patients with osmophobia were more likely to have higher level of depression and anxiety than those without osmophobia [7]. In addition, patients with allodynia had a higher frequency of anxiety thacn those without allodynia and those with moderate to severe allodynia had a higher risk of developing depression than those with no or mild allodynia [11].

Despite the significance of migraine with aura for suicidality, relevant studies did not consider sensory hypersensitivities as risk factors of suicide despite the existence of the relationship between sensory hypersensitivities and psychiatric symptoms [7, 11]. Therefore, we investigated whether sensory hypersensitivities contributed to suicidality in patients with migraine.

\section{苗}

(c) 2015 Park et al.; licensee Springer. This is an Open Access article distributed under the terms of the Creative Commons Attribution License (http://creativecommons.org/licenses/by/4.0), which permits unrestricted use, distribution, and reproduction in any medium, provided the original work is properly credited. 


\section{Methods}

\section{Subjects}

We invited new patients with migraine who had consecutively visited a headache clinic in the Department of Neurology at Kyungpook National University Hospital since September 2013. Patients were adolescents and adults between the ages of 15 and 75 . They were newly diagnosed patients at the visit or already diagnosed patients but did not take preventive medicines for migraine and other psychotropic agents within a month. A diagnosis of migraine was assigned based on the International Classification of Headache Disorders, 3rd edition, beta version (ICHD-3 beta) by a trained neurologist (SP Park) [13]. Patients who had illiteracy, mental retardation, serious medical, neurological, or psychiatric disorders, and alcohol or drug abuse that prevented them from cooperating in the psychiatric interview and understanding the questionnaire were excluded. Patients who had a probable migraine or who declined to participate in the interview were also excluded.

\section{Study design}

A cross-sectional study was conducted as part of a hospital-based study which examined the impact of psychiatric disorders on migraine. The Institutional Review Board of Kyungpook National University Hospital approved the study. All participants obtained written informed consent. SP Park interviewed each patient and reviewed the patient's medical charts to collect demographic and clinical information for a computerized database. Sociodemographic data were collected on variables including age, gender, education, place of residence (city or country), religion, employment, household income (earning at least three million KRW per month, equivalent to 2800 USD per month or not), and marital status (married or unmarried, divorced, and bereaved). Clinical data included; type of migraine, migraine chronicity (episodic migraine $[\mathrm{EM}]$ or chronic migraine $[\mathrm{CM}]$ ), medication overuse headache $(\mathrm{MOH})$, age at onset, disease duration, headache intensity, accompanying symptoms (presence of nausea and/or vomiting, photophobia, phonophobia, osmophobia, or allodynia) and family history. The Visual Analog Scale (VAS) measured headache intensity. The VAS was measured in two different ways, i.e. the $\mathrm{VAS}_{\max }$ and $\mathrm{VAS}_{\text {now }} \mathrm{VAS}_{\text {max }}$ meant the maximal intensity of headaches experienced during the prior three months, and $\mathrm{VAS}_{\text {now }}$ represented the intensity of the headache on the day of the psychiatric interviews. Photophobia, phonophobia, and osmophobia were defined as the hypersensitivity to light, sound, and certain odors during migraine attacks which could cause avoidance of those stimulations or aggravation of migraine symptoms. We asked patients whether they experienced symptoms in the preceding year. Allodynia was measured using the 12-item Allodynia
Symptom Checklist (ASC-12) [14] with a cut-off score of $>2$ defining allodynic patients. Family history of migraine was defined as the existence of migraine diagnosis in a lineal ascendant and siblings. Migraine disability was measured by the Migraine Disability Assessment Scale (MIDAS) [15]. The overall level of disability was represented as follows; grade I, little or no disability (score of $0-5)$, grade II, mild disability (6-10), grade III, moderate disability (11-20), and grade IV, severe disability (21 or more). Disability of grade I and II was compared to that of grade III and IV.

\section{Psychiatric interviews}

All participants were interviewed by a neuropsychologist within a week after the first visit to determine whether they had current major depressive disorder (MDD), current generalized anxiety disorder (GAD) or suicidality using the Korean version of the Mini-International Neuropsychiatric Interview-Plus 5.0.0. (MINI) [16]. The MINI is a brief, structured interview based on DSM-IV criteria [17], and is recommended to screen for psychiatric comorbidity in headache patients [18]. The Kappa values of MDD and GAD in the Korean version were 0.71 and 0.57 , respectively, which exhibited good agreement between the MINI and the expert's diagnosis. The suicidality module is composed of six questions of different weight, including five questions asking about current suicidality (which includes suicidal ideation or attempt in the preceding month: wish for death [weight 1], wish for self-harm [weight 2], suicidal thought [weight 6], suicide plan [weight 10], and suicide attempt [weight 10], and one question asking the lifetime suicide attempt [weight 4]). If respondents said "yes" for at least one of the six questions, they were thought to have suicidality. The degree of current suicidality is estimated from the sum of the weighted score of the six questions, with low $(1-5)$, moderate (6-9), and high $(\geq 10)$ level of suicide risk.

\section{Statistical analyses}

The Statistical Package for the Social Sciences (SPSS version 19.0) was used for the analyses of data. Descriptive statistics are presented in terms of counts, percentages, means, and standard deviations. To determine factors for suicidality, a logistic regression model with suicidality as the outcome was developed, using a two-step procedure. First, univariate logistic regression analyses were used to test which potential variables were associated with suicidality among patients with migraine. Second, all variables with a statistically significant univariate effect were entered in a multivariate logistic regression model and a forward likelihood ratio test was chosen to assess the differential contribution of individual variables to suicidality. Results were reported as odds ratio (OR) 
with $95 \%$ confidence intervals, standardized regression coefficients (Beta) and corresponding $p$ values. The level of statistical significance was set at $p<0.05$.

\section{Results}

A total of 254 patients with migraine consecutively visited our headache clinic. Among them, 34 patients were excluded because of refusal to interview $(n=12)$, probable migraine $(\mathrm{n}=10)$, taking preventive medicine within a month $(n=4)$, age older than $75(n=4)$, mental retardation $(n=2)$, or illiteracy $(n=2)$. Subsequently, 220 patients (30 men/190 women, mean age 40.1 \pm 13.1$)$ completed the study. Among them, 17 patients (7.7 \%) had migraine with aura and 203 patients (92.3\%) had migraine without aura. EM was manifested in 95 patients (43.2 \%), CM in 125 patients (56.8\%), and $\mathrm{MOH}$ in 52 patients $(23.6 \%)$. All patients with $\mathrm{MOH}$ had CM. Photophobia, phonophobia, osmophobia, and allodynia were manifested in 104 patients (47.3\%), 140 patients (63.6\%), 108 patients (49.1\%), and 39 patients (17.7\%), respectively. Current MDD and current GAD were revealed in 56 patients $(25.5 \%)$ and 38 patients $(17.3 \%)$, respectively. Suicidality was documented in 70 patients (31.8\%). Among them, 25 patients (35.7 \%) had a moderate to high degree of suicidality. Current suicidality, lifetime suicide attempt, or both were revealed in 38 patients (54.3\%), 18 patients $(25.7 \%)$, and 14 patients $(20 \%)$, respectively. Forty patients $(57.1 \%)$ had only suicidal ideation and 30 patients $(42.9 \%)$ had current or lifetime suicidal attempt.

Sociodemographic, clinical, and psychiatric aspects of migraine with respect to suicidality are listed in Table 1. Factors contributing to suicidality by univariate analyses are documented in Table 2 . Patients with suicidality were more like to have a low household income (OR 1.88, $95 \%$ confidence interval $[\mathrm{CI}]$ 1.04-3.41, $p=0.038), \mathrm{CM}$ (OR 3.05, 95 \% CI 1.63-5.69, $p<0.001$ ), MOH (OR 2.27, $95 \%$ CI 1.20-4.32, $p=0.012$ ), high $\mathrm{VAS}_{\max }$ score (OR 0.80, $95 \%$ CI 0.68-0.94, $p=0.007)$, high $\mathrm{VAS}_{\text {now }}$ score (OR 0.81, 95 \% CI 0.72-0.91, $p<0.001$ ), osmophobia (OR 2.98, $95 \%$ CI 1.64-5.41, $p<0.001$ ), allodynia (OR 4.70, $95 \%$ CI 2.27-9.71, $p<0.001$ ), high MIDAS grade (OR 3.05, $95 \%$ CI 1.63-5.69, $p<0.001$ ), current MDD (OR 5.50, $95 \%$ CI 2.87-10.54, $p<0.001$ ), and current GAD (OR 5.86, $95 \%$ CI 2.77-12.40, $p<0.001$ ) than those without suicidality. Age, gender, education, and the type of migraine were not associated with suicidality. The frequency of osmophobia or allodynia was not significantly changed by the degree of suicidality or if patients had only suicidal ideation or attempted suicide. In addition, it was not affected by if patients had current suicidality or lifetime suicidal attempts.

Risk factors contributing to suicidality by multivariate analyses are documented in Table 3 . The strongest risk factor for suicidality was osmophobia (Beta 0.314, adjusted
OR [AOR] 3.12, $95 \%$ CI 1.57-6.21, $p=0.001$ ), followed by current MDD (Beta 0.264, AOR 2.99, $95 \%$ CI 1.336.76, $p=0.008$ ), CM (Beta 0.249, AOR 2.48, $95 \%$ CI 1.21-5.09, $p=0.013$ ), current GAD (Beta, 0.237, AOR 3.11, $95 \%$ CI 1.22-7.91, $p=0.017$ ), and allodynia (Beta 0.211, AOR 2.72, $95 \%$ CI 1.19-6.21, $p=0.018$ ). A forward likelihood ratio test produced a five-variable model that explained $34.3 \%$ of the variance in suicidality. According to Beta, the contribution of osmophobia to suicidality was 1.19 times greater than that of current MDD, 1.26 times greater than that of CM, 1.32 times greater than that of current GAD, and 1.49 times greater than that of allodynia.

\section{Discussion}

Sensory hypersensitivities such as photophobia and phonophobia were frequently observed in patients with migraine and included in the diagnostic criteria of migraine of the International Classification of Headache Disorders (ICHD)-3 beta [13]. The other types of hypersensitivities, osmophobia and allodynia, were also frequently manifested in patients with migraine [7-12], but were not included in the diagnostic criteria of migraine. Although osmophobia has been reported as one of the specific symptoms for differentiating migraine from other headache disorders [7-9], it remains underrecognized as a migraine-associated symptom, and its clinical implications are under-investigated [7]. Under these circumstances, we found that osmophobia and allodynia were major risk factors for suicidality in patients with migraine, after controlling for MDD, GAD, and CM.

Regarding risk factors for suicidality in patients with migraine, several studies reported psychiatric disorders, sociodemographic factors, type of migraine, headache frequency and intensity, and migraine disability [1-6]. We also found similar results in univariate analyses. However, sensory hypersensitivities, such as osmophobia and allodynia, were found to be critical factors by multivariate analyses. We also found that the contribution of osmophobia to suicidality is a little higher and the contribution of allodynia to suicidality is a little lower than that of depression or anxiety. This means that osmophobia and allodynia are important to determine suicidality as much as psychiatric disorders.

The mechanism to explain the contribution of osmophobia to suicidality is unknown. One possibility is a common pathway of osmophobia and mood changes may provoke suicidality. Smell is innately related to the limbic system. In an event-related fMRI study, olfactory stimulation during migraine attack produced increased limbic and brainstem activity [19]. In mood disorders, several limbic areas have been reported to be altered in structural, functional and molecular neuroimaging [20]. Therefore, altered function of the limbic system appears 
to produce psychiatric disorders as well as osmophobia, and finally can provoke suicidality. However, this theory may be insufficient to explain the mechanism in our patients, because osmophobia can contribute to suicidality regardless of the psychiatric disorders. Another possibility is osmophobia is associated with headache intensity, and severe pain accompanying osmophobia may provoke suicidality. In a population-based study, headache severity at baseline predicted suicide attempts during the two-year follow-up period in migraine or non-migraine headache [4]. In a hospital-based study, headache intensity was a risk factor for suicidal ideation in patients with migraine after controlling for depression [6]. Through these results, if osmophobia is an expression for severe pain, it can be a risk factor for suicidality. Further studies are needed to elucidate these relationships. The

Table 1 Sociodemographic, clinical, and psychiatric aspects of eligible subjects ( $n=220$ ) with respect to suicidality

\begin{tabular}{|c|c|c|}
\hline \multirow[b]{3}{*}{ Characteristic } & \multicolumn{2}{|c|}{ Mean \pm SD (range) or number (\%) } \\
\hline & Suicidality & No suicidality \\
\hline & $(n=70)$ & $(n=150)$ \\
\hline Age, years & $40.3 \pm 13.2(16-73)$ & $40.0 \pm 13.0(15-65)$ \\
\hline Gender, female & $62(88.6)$ & $128(85.3)$ \\
\hline Education, years & $12.4 \pm 2.9(6-18)$ & $13.0 \pm 2.8(5-18)$ \\
\hline Place of residence, city & $61(87.1)$ & $125(83.3)$ \\
\hline Religion, yes & $36(51.4)$ & $81(54.0)$ \\
\hline Employment, yes & $34(48.6)$ & $72(48.0)$ \\
\hline Household income, at least 3 million KRW/month & $41(58.6)$ & $109(72.7)$ \\
\hline Marital status, no history of divorce or bereavement & $42(60.0)$ & $95(63.3)$ \\
\hline \multicolumn{3}{|l|}{ Type of migraine } \\
\hline Migraine with aura & $4(5.7)$ & $13(8.7)$ \\
\hline Migraine without aura & $66(94.3)$ & $137(91.3)$ \\
\hline \multicolumn{3}{|l|}{ Migraine chronicity } \\
\hline Episodic migraine & $18(25.7)$ & $77(51.3)$ \\
\hline Chronic migraine & $52(74.3)$ & $73(48.7)$ \\
\hline Family history of migraine & $45(64.3)$ & $96(64.0)$ \\
\hline $\mathrm{MOH}$ & $24(34.3)$ & $28(18.7)$ \\
\hline Age of onset & $29.7 \pm 12.0(9-53)$ & $30.5 \pm 12.3(8-59)$ \\
\hline Disease duration & $10.6 \pm 9.4(0.3-36)$ & $9.4 \pm 7.3(0.2-37)$ \\
\hline$V A S_{\max }^{a}$ & $8.5 \pm 1.5(3-10)$ & $7.7 \pm 2.2(0-10)$ \\
\hline VAS now & $3.2 \pm 2.6(0-10)$ & $1.9 \pm 2.3(0-8)$ \\
\hline \multicolumn{3}{|l|}{ Accompaning symptoms } \\
\hline Nausea and/or vomiting & $60(85.7)$ & $132(88.0)$ \\
\hline Photophobia & $33(47.1)$ & $71(47.3)$ \\
\hline Phonophobia & $51(72.9)$ & $89(59.3)$ \\
\hline Osmophobia & $47(67.1)$ & $61(40.7)$ \\
\hline Allodynia & $24(34.3)$ & $15(10.0)$ \\
\hline MIDAS score & $42.0 \pm 41.4(0-190)$ & $18.3 \pm 21.9(0-135)$ \\
\hline \multicolumn{3}{|l|}{ MIDAS grade } \\
\hline Grade I and II & $18(25.7)$ & $77(51.3)$ \\
\hline Grade III and IV & $52(74.3)$ & $73(48.7)$ \\
\hline Current MDD & $34(48.6)$ & $22(14.7)$ \\
\hline Current GAD & $25(35.7)$ & $13(8.7)$ \\
\hline
\end{tabular}

Abbreviations: $\mathrm{KRW}=$ Korean Won; $\mathrm{MOH}=$ medication overuse headache; VAS = Visual Analog Scale, MIDAS = Migraine Disability Assessment Scale; MDD = major depressive disorder; GAD = generalized anxiety disorder

${ }^{a}$ Maximal headache intensity during the preceding 3 months

${ }^{\mathrm{b}}$ Headache intensity on the day of psychiatric interviews 
Table 2 Factors contributing to suicidality by univariate analyses

\begin{tabular}{|c|c|c|c|c|}
\hline Variable & $\beta$ & $\mathrm{SE}(\beta)$ & OR $(95 \% \mathrm{Cl})$ & $p$ value \\
\hline Age & -0.002 & 0.011 & $1.00(0.98-1.02)$ & 0.862 \\
\hline Gender & 0.287 & 0.441 & $1.33(0.56-3.16)$ & 0.516 \\
\hline Education & 0.076 & 0.051 & $1.08(0.98-1.19)$ & 0.134 \\
\hline Place of residence & 0.304 & 0.419 & $1.36(0.60-3.08)$ & 0.468 \\
\hline Religion & -0.103 & 0.29 & $0.90(0.51-1.59)$ & 0.722 \\
\hline Employment & 0.023 & 0.29 & $1.02(0.58-1.81)$ & 0.937 \\
\hline Household income & 0.631 & 0.304 & $1.88(1.04-3.41)$ & 0.038 \\
\hline Marital status & 0.141 & 0.297 & $1.15(0.64-2.06)$ & 0.635 \\
\hline Type of migraine & -0.448 & 0.591 & $0.64(0.20-2.03)$ & 0.448 \\
\hline CM & 1.114 & 0.319 & $3.05(1.63-5.69)$ & $<0.001$ \\
\hline Family history of migraine & 0.012 & 0.302 & $1.01(0.56-1.83)$ & 0.967 \\
\hline $\mathrm{MOH}$ & 0.821 & 0.328 & $2.27(1.20-4.32)$ & 0.012 \\
\hline Age at onset & 0.006 & 0.012 & $1.01(0.98-1.03)$ & 0.640 \\
\hline Disease duration & -0.018 & 0.018 & $0.98(0.95-1.02)$ & 0.304 \\
\hline$V A S_{\max }^{a}$ & -0.221 & 0.082 & $0.80(0.68-0.94)$ & 0.007 \\
\hline$V A S_{\text {now }}^{b}$ & -0.208 & 0.059 & $0.81(0.72-0.91)$ & $<0.001$ \\
\hline Nausea and/or vomiting & -0.201 & 0.424 & $0.82(0.36-1.88)$ & 0.636 \\
\hline Photophobia & -0.008 & 0.290 & $0.99(0.56-1.75)$ & 0.979 \\
\hline Phonophobia & 0.610 & 0.316 & $1.84(0.99-3.42)$ & 0.054 \\
\hline Osmophobia & 1.092 & 0.304 & $2.98(1.64-5.41)$ & $<0.001$ \\
\hline Allodynia & 1.547 & 0.371 & $4.70(2.27-9.71)$ & $<0.001$ \\
\hline MIDAS grade & 1.114 & 0.319 & $3.05(1.63-5.69)$ & $<0.001$ \\
\hline Current MDD & 1.704 & 0.332 & $5.50(2.87-10.54)$ & $<0.001$ \\
\hline Current GAD & 1.767 & 0.383 & $5.86(2.77-12.40)$ & $<0.001$ \\
\hline
\end{tabular}

Abbreviations: $\mathrm{CM}=$ chronic migraine; $\mathrm{MOH}=$ medication overuse headache; VAS = Visual Analog Scale, MIDAS = Migraine Disability Assessment Scale; $\mathrm{MDD}=$ major depressive disorder $\mathrm{GAD}=$ generalized anxiety disorder ${ }^{a}$ Maximal headache intensity during the preceding 3 months

${ }^{b}$ Headache intensity on the day of psychiatric interviews

Mechanism for the impact of allodynia to suicidality is a little easier to explain. Patients with allodynia can reveal suicidality not only by a high frequency of comorbid depression and anxiety $[11,12]$ but also by a high probability of migraine chronification [10]. Of course, allodynia

Table 3 Factors contributing to suicidality by multivariate analyses

\begin{tabular}{llllll}
\hline Variable & $\beta$ & SE $(\beta)$ & Beta $^{a}$ & Adjusted OR (95 \% Cl) & $p$ value \\
\hline Constant & -2.577 & 0.547 & & 0.08 & $<0.001$ \\
Osmophobia & 1.138 & 0.351 & 0.314 & $3.12(1.57-6.21)$ & 0.001 \\
Current MDD & 1.096 & 0.416 & 0.264 & $2.99(1.33-6.76)$ & 0.008 \\
CM & 0.909 & 0.367 & 0.249 & $2.48(1.21-5.09)$ & 0.013 \\
Current GAD & 1.134 & 0.476 & 0.237 & $3.11(1.22-7.91)$ & 0.017 \\
Allodynia & 1.000 & 0.422 & 0.211 & $2.72(1.19-6.21)$ & 0.018 \\
\hline
\end{tabular}

Abbreviations: $\mathrm{MDD}=$ major depressive disorder; $\mathrm{CM}=$ chronic migraine; $\mathrm{GAD}=$ generalized anxiety disorder

${ }^{\text {a }}$ Standardized regression coefficients may also be an expression for severe pain. The relationship of them should be clarified.

Our study failed to detect that migraine with aura was an important risk factor for suicidality. There may be several reasons to explain such. First, relevant studies were population-based studies with a large sample sizes [1-3]. On the other hand, our study was a hospitalbased study, and the number of patients who had migraine with aura was 17 . Therefore, the selection bias may affect the result. Second, previous studies were conducted in adolescents (12-15 years old) [2,3] or young adults (21-30 years old) [1] with migraine, but not in patients of all ages. We investigated risk factors of suicidality in patients who were 15 to 73 years old (mean 40.1 years old). Therefore, the different age groups of eligible patients can elicit different results. In a large sample, community-based study, migraine with aura was not a risk factor for suicidal attempt in patients who were 25 to 55 years old (mean 40.4 years old) [4]. A further study to investigate risk factors according to the different age groups should be needed. Third, they did not consider the impact of sensory hypersensitivities on suicidality [1-3] despite the existence of a relationship between osmophobia or allodynia and psychiatric symptoms [7, 11]. Fourth, they did not investigate the impact of $C M$ in relation to episodic migraine (EM) on suicide [1-3]. As expected, CM results in substantially greater disability than EM. In a hospital-based study in Korea, CM sufferers were more likely to have depression, anxiety, disability, and impaired quality of life than EM sufferers [12]. In a review of the literatures, authors summarized that patients with chronic daily headache such as CM and chronic tension-type headache appears to have a high suicidal intent due to depression, disability, impaired quality of life and chronic pain [21]. Therefore, CM should be included as a variable to determine suicide. After all, if we investigate risk factors for suicidality in patients with migraine considering these issues, more precise data will be obtained.

Our study has some limitations. First, this is a hospitalbased study, which may not represent the whole migraine population. Therefore, a large sample, population-based study should be done. Second, the cross-sectional nature of our study prevented us from drawing conclusions about the causal relationship between osmophobia or allodynia and suicidality. A longitudinal study is needed. Third, ethnic differences could also limit the generalization of our results, for example, our patients revealed a higher frequency of osmophobia and a lower frequency of allodynia than Western patients did. Further studies to prove our results should also be conducted in Western countries. Fourth, we did not measure olfactory function during the ictal period of the migraine. Subjective feelings and objective findings of olfaction may draw different conclusions. 
Fifth, we did not investigate the impact of comorbid organic diseases on suicidality. However, we excluded patients who had serious medical, neurological, or psychiatric disorders from the study, so the impact of other organic diseases on suicidality is likely to be trivial.

\section{Conclusions}

Suicide is the tenth leading cause of death worldwide [22] with about 800,000 to one million people dying annually, giving a mortality rate of 11.6 per 100,000 persons per year [23]. In a two year population-based observational study, suicidal attempt is 4.43 times higher in patients with migraine than in healthy controls when sex, psychiatric disorder, and previous history of suicidal attempts had been adjusted for at baseline [4]. Almost $32 \%$ of our patients manifested suicidality and nearly $43 \%$ of them attempted suicide when they visited a tertiary care hospital. Under these circumstances, clinicians should always observe carefully the existence of suicidality in patients with migraine who visit outpatient clinics. In a busy clinical setting, psychiatric interviews, including suicidality, take a long time to conduct. In this situation, simply asking the patients about osmophobia or allodynia will be an initial step for further evaluation of suicidality. A global study to evaluate sensory hypersensitivities as a red flag for suicidality should be done in the future.

\section{Abbreviations \\ ICHD-3: International Classification of Headache Disorders; EM: Episodic migraine; CM: Chronic migraine; $\mathrm{MOH}$ : Medication overuse headache; VAS: Visual Analog Scale; ASC: Allodynia Symptom Checklist; MIDAS: Migraine Disability Assessment Scale; MDD: Major depressive disorder; \\ GAD: Generalized anxiety disorder; MINI: Mini-International Neuropsychiatric Interview; OR: Odd Ratio.}

\section{Competing interests}

The authors declare that they have no competing interests.

\section{Authors' contributions}

SPP took part in the design of the study, contributed to the data collection. SPP and JGS participated in writing the manuscript. SPP and WKL was responsible for data statistics. All authors agreed to accept equal responsibility for the accuracy of the content of the paper. All authors read and approved the final manuscript.

\section{Acknowledgements}

The authors thank Ju-Hui Lee, a neuropsychologist, for conducting the MINI-Plus 5.0.0 and helping in the completion of self-report questionnaires.

\section{Funding}

This research received no specific grant from any funding agency in the public, commercial, or not-for-profit sectors.

\section{Author details}

'Department of Neurology, School of Medicine, Kyungpook National University, 680 Gukchaebosang-ro, Jung-gu, Daegu 700-842, Republic of Korea. ${ }^{2}$ Center of Biostatics, School of Medicine, Kyungpook National University, Daegu, Republic of Korea.

\section{References}

1. Breslau N (1992) Migraine, suicidal ideation, and suicide attempts. Neurology 42:392-395

2. Wang SJ, Juang KD, Fuh JL, Lu SR (2007) Psychiatric comorbidity and suicide risk in adolescents with chronic daily headache. Neurology 68:1468-1473

3. Wang SJ, Fuh JL, Juang KD, Lu SR (2009) Migraine and suicidal ideation in adolescents aged 13 to 15 years. Neurology 72:1146-1152

4. Breslau N, Schultz L, Lipton R, Peterson E, Welch KM (2012) Migraine headaches and suicide attempt. Headache 52:723-731

5. Fuller-Thomson E, Schrumm M, Brennenstuhl S (2013) Migraine and despair: factors associated with depression and suicidal ideation among Canadian migraineurs in a population-based study. Depress Res Treat 2013:401487

6. Kim SY, Park SP (2014) Suicidal ideation and risk factors in Korean migraine patients. J Clin Neurosci 21:1699-1704

7. Wang YF, Fuh JL, Chen SP, Wu JC, Wang SJ (2012) Clinical correlates and diagnostic utility of osmophobia in migraine. Cephalalgia 32:1180-1188

8. Silva-Néto RP, Peres MF, Valença MM (2014) Accuracy of osmophobia in the differential diagnosis between migraine and tension-type headache. J Neurol Sci 339:118-122

9. Zanchin G, Dainese F, Trucco M, Mainardi F, Mampreso E, Maggioni F (2007) Osmophobia in migraine and tension-type headache and its clinical features in patients with migraine. Cephalalgia 27:1061-1068

10. Louter MA, Bosker JE, van Oosterhout WP, van Zwet EW, Zitman FG, Ferrari MD et al (2013) Cutaneous allodynia as a predictor of migraine chronification. Brain 136:3489-3496

11. Kao CH, Wang SJ, Tsai CF, Chen SP, Wang YF, Fuh JL (2014) Psychiatric comorbidities in allodynic migraineurs. Cephalalgia 34:211-218

12. Kim SY, Park SP (2014) The role of headache chronicity among predictors contributing to quality of life in patients with migraine: a hospital-based study. J Headache Pain 15:68

13. Headache Classification Committee of the International Headache Society (IHS) (2013) The International classification of headache disorders, 3rd edition (beta version). Cephalalgia 33:629-808

14. Lipton RB, Bigal ME, Ashina S, Burstein R, Silberstein S, Reed ML, Serrano D, Stewart WF; American Migraine Prevalence Prevention Advisory Group (2008) Cutaneous allodynia in the migraine population. Ann Neurol 63:148-158

15. Lee HS, Chung CS, Song HJ, Park HS (2000) The reliability and validity of the MIDAS (Migraine Disability Assessment) Questionnaire for Korean migraine sufferers. J Korean Neurol Assoc 18:287-291

16. Yoo SW, Kim YS, Noh JS, Oh KS, Kim CH, Namkoong K et al (2006) Validity of Korean version of the MINI-international neuropsychiatric interview. Anxiety and Mood 2:50-55

17. Sheehan DV, Lecrubier $Y$, Sheehan $\mathrm{KH}$, Amorim $\mathrm{P}$, Janavs J, Weiller $E$ et al (1998) The Mini-International Neuropsychiatric Interview (M.I.N.I.): the development and validation of a structured diagnostic psychiatric interview for DSM-IV and ICD-10. J Clin Psychiatry 59(suppl 20):S22-S33

18. Maizels M, Smitherman TA, Penzien DB (2006) A review of screening tools for psychiatric comorbidity in headache patients. Headache 46(suppl 3):S98-\$109

19. Stankewitz A, May A (2011) Increased limbic and brainstem activity during migraine attacks following olfactory stimulation. Neurology 77:476-482

20. Höflich A, Baldinger P, Savli M, Lanzenberger R, Kasper S (2012) Imaging treatment effects in depression. Rev Neurosci 23:227-252

21. Pompili M, Di Cosimo D, Innamorati M, Lester D, Tatarelli R, Martelletti P (2009) Psychiatric comorbidity in patients with chronic daily headache and migraine: a selective overview including personality traits and suicide risk. J Headache Pain 10:283-290

22. Hawton K, van Heeringen K (2009) Suicide. Lancet 373:1372-1381

23. Värnik P (2012) Suicide in the world. Int J Environ Res Public Health 9:760-771 